\title{
12th Annual University of Wisconsin Land O'Lakes Bioanalytical Conference
}

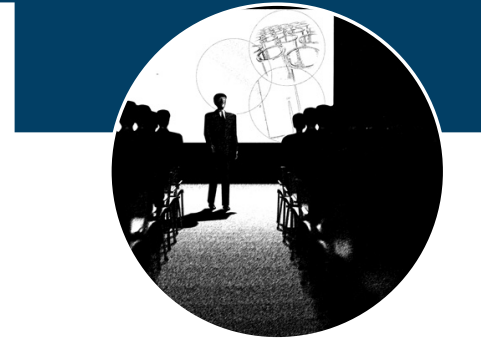

\author{
I2th Annual Land O'Lakes Bioanalytical Conference: Agency and Industry Perspectives on \\ Biomarkers, Bloodspots and Beyond \\ Devil's Head Resort, Merrimac, WI, USA, II-15 July 201 I
}

This University of Wisconsin School of Pharmacy bioanalytical conference is presented each year by the Extension Services in Pharmacy, the professional development department within the school. The purpose of this 4-day conference is to provide an educational forum to discuss issues and applications associated with the analysis of xenobiotics, metabolites, biologics and biomarkers in biological matrices. The conference is designed to include and encourage an open exchange of scientific and methodological applications for bioanalysis. To increase the interactive nature of the conference, the program was a mixture of lectures, poster sessions, round table discussions and workshops. This article summarizes the presentations at the 12th Annual Conference.

The 12th Annual Land O'Lakes Bioanalytical Conference, titled 'Agency and Industry Perspectives on Biomarkers, Bloodspots and Beyond', was held on 11-15 July 2011 at Devil's Head Resort in Merrimac, WI, USA. This conference brings together an international group of bioanalytical scientists with the express intent of discussing cutting-edge science for small- and large-molecule bioanalysis in a relaxed atmosphere that also promotes networking opportunities. The conference provides an educational forum with presentations by scientific leaders from academia, industry, contract laboratories and regulatory authorities. The success of the program is due to the work of the Planning Committee, which consists of scientists involved with bioanalysis on a daily basis, and their ability to identify timely topics and presenters who are experts in the field. Specific objectives for the conference and a list of the Planning Committee members can be found at $[1]$.

\section{Four plenary sessions}

Morning meetings consisted of four plenary sessions focusing on specific themes associated with bioanalytical issues. The first plenary session was titled 'Biomarkers and diagnostic kits (large- and small-molecule applications)'. Hawazin Faruki (LabCorp) provided the opening overview of the topic and set the stage with examples of critical biomarkers recently identified and effectively validated for use in disease monitoring. She highlighted the challenges of identifying and using biomarkers in clinical trials, including poor biomarker performance, inability to demonstrate clinical utility, limited availability of specimens, regulatory uncertainty, high costs of the tests and uncertain returns on investment for profit on testing. Once tests are cleared by the US FDA they are prevalidated and generally more easily obtained, leading to more widespread use. Research kits are more variable and can be critical for use in clinical trials. Modification and adjustment of those kits may be necessary. The second presentation by Joe Palandra (Pfizer) discussed efforts at quantitating protein biomarkers using LC-MS/ MS techniques. MS is a good approach for the detection of protein biomarkers in early research because it can match or exceed the sensitivity of immunoassays while requiring fewer antibodies for protein or peptide enrichment. His group is exploring multiple approaches to protein quantitation using various immunoaffinity methods including bead-based, column-based, protein enrichment and peptide enrichment methods. In all cases, digestion is required since intact proteins are too large for MS quantitation. It is also necessary to identify the best way to incorporate internal standards. There are many approaches in the literature and his group is typically using one of the stable label approaches depending on the application. Lauren Stevenson (Biogen Idec) then talked about her company's use of standard immunoassays and adapted kits in biomarker assay development. She used examples from her group's research to demonstrate those challenges, which included lack of available reference standards, limited availability/quality of reagents and issues with commercially available kits. She expanded on the issues with kits, including the lack of sensitivity, too few quality
James E DeMuth ${ }^{\dagger 1}$, Chad Briscoe $^{2}$, Lakshmi

Amaravadi ${ }^{3}$, Mark E Arnold $^{4}$, Robert P Clement $^{5}$, Eric N Fluhler ${ }^{6}$, Qin C Ji \& R John Stubbs ${ }^{8}$

'University of Wisconsin, 777 Highland Ave., Madison, WI 53705, USA

2PRA International, Lenexa, KS,

USA

${ }^{3}$ Biogen Idec, Cambridge, MA, USA ${ }^{4}$ Bristol-Myers Squibb Company,

New Brunswick, NJ, USA

${ }^{5}$ Merck Research Laboratories, Summit, NJ, USA

${ }^{6}$ Pfizer Research, Groton, CT, USA ${ }^{7}$ Bristol-Myers Squibb Company,

New Brunswick, NJ, USA

${ }^{8}$ Stubbs and Hensel Pharma Consulting, LLC, Blue Bell, PA, USA

${ }^{\dagger}$ Author for correspondence:

E-mail: jedemuth@

pharmacy.wisc.edu 
controls/calibrators, as well as changes and lack of reliability in kit supply from manufactures. She used examples from her research to demonstrate ways to overcome these challenges. The final talk for the first plenary session was from Brian Booth (CDER, US FDA) who gave an overview and several examples of biomarkers being used successfully in clinical trial submissions. Biomarkers can be measured in many ways for analytical tests from imaging to molecular assays. For exploratory biomarkers one should employ an appropriate amount of validation, but for biomarkers intended for dosing, drug approval, or labeling, full validation is required as per the current pharmacokinetic (PK)-based criteria described in the FDA's 'Guidance for Bioanalytical Method Validation.'

Wednesday's plenary session addressed emerging analytical issues related to measurement of large-molecule therapeutics. Priya Sriraman (Roche) gave an overview of the current status of clinical development of bispecific antibodies, covered structural features of bispecifics and other related molecules such as BiTES, chemically generated scaffold antibodies (e.g., Cov-x bodies), bispecifics using hybrid hybridomas (quadromas), tri-functional bispecifics (TRION), tandem Sc-Fvs, diabodies and others. The talk addressed various structural features of these new generation molecules, functional advantages offered in vivo, their PK properties and analytical challenges. Unique analytical issues such as intact drug versus individual components of the drug that may be present in circulation, considerations related to identification of relevant molecules for measurement, characterization assays applicable in early development and the challenges related to the specificity of antidrug antibodies and bispecifics were highlighted. Christopher Stebbins (Biogen Idec) then presented a detailed case study example of a bispecific antibody that binds to two different ligands. He described the analytical challenges related to PK assay development, covered three different assay formats used in the research phase of the drug development, and highlighted the importance of the generation of appropriate reagents early in development. In one case study, findings were related to the short half-life of the molecule (2-4 days) in cynomolgus monkey. He discussed possible factors that could lead to observed half-lives (such as failure to bind to the neonatal Fc receptor for IgG [FcRn], lack of stability, ligand interference and immunogenicity) and eliminated some of these based on experimental evaluation. He also discussed analytical challenges related to immunogenicity assays, such as ligand interference leading to false-positive results. Renuka Pillutla (BristolMyers Squibb) discussed analytical issues related to larger molecules, including interference from circulating targets. Her presentation focused on quantitation of large molecules, ligand-binding assays, possible interferences and bioanalytical considerations with respect to binding affinities and assay formats. She concluded with a case study using appropriate assay formats for measuring free and total drug concentrations. Timothy Olah (Bristol-Myers Squibb) concluded the plenary session with a presentation on LC-MS-based method development for the quantitation of therapeutic proteins in plasma. He focused on establishing multiple component bioanalytical strategies that support discovery biologics, how to determine how best to implement current technologies that simultaneously quantify multiple components and how to establish practices to better understand and interpret complementary data generated in multiple component analysis.

The Thursday morning plenary session focused on the current state-of-the-art for dried blood spots (DBS) and related microsampling techniques. Christopher Evans (GlaxoSmithKline) started the session with a presentation that provided the recent history and data leading up to the present. In-depth experimentation across a broad spectrum of the industry, academic and CRO laboratories has identified the strengths of DBS, as well as many of its limitations. He discussed some recent work that showed trends in drug concentrations across spots of varying plasma protein (e.g., albumin). Evans also noted other microsampling techniques and foretold a bit of the future for DBS. Shane Needham (Alturus Analytics) spoke on approaches to validating DBS methods. Similar to the collection of blood, its centrifugation and then frozen storage as plasma, DBS is nothing more than another sample collection and storage technique that provides a dried sample matrix. His talk covered the fundamentals of critical parameters for solvating the spots punched from the card as part of the extraction procedure and the several approaches used for internal standard addition. Needham presented two of the most common approaches to achieve sample solutions for samples whose concentrations exceed the upper limit of quantitation. He reviewed the approaches possible for determining stability and recovery, key aspects of a rugged method and pointed out a variety of situations where stability 
was not always easily obtained, including one case where samples did require frozen storage. A number of case studies involving method development and use of DBS were next presented by Ed Brewer (Tandem Labs). He highlighted the key parameters of method development and validation that needed to be assessed for DBS through comparisons of validation data for a compound in plasma and on DBS cards. He cited specific examples of where treating the stock DBS cards with acids was required to stabilize some analytes, noting that stability has two phases (during the drying process and then during storage) and how each must be examined. He closed by noting how simple it is to use liquid handlers to add stabilizers to create custom-treated cards for laboratory experimentation. The closing presentation was made by CT Viswanathan (CDER, US FDA). As part of the FDA's Division of Safety Inspections, he focused on some on the Agency's current thinking on DBS and microsampling techniques. The Agency was very interested in DBS and micro sampling for the advantages it provides, but was clear that the Agency lacks enough information to develop a definitive position. The Agency was pleased to see the indepth characterization that the industry has undertaken to understand the strengths and weaknesses associated with DBS. It is up to the sponsors to provide compelling evidence of the reliability of the technique as part of any filing and that its use had to fit the intended purpose. He presented this fit-forpurpose approach linked to the depth of the validation efforts needed as a continuum from animal PK studies, into good laboratory practice safety studies in animal, to early human studies concluding in the highest value. Viswanathan expressed extreme caution to anyone who uses DBS for bioequivalence studies and made clear that any sponsor could at any time request a meeting with the Agency to discuss their plans for using DBS.

The Friday morning plenary session featured two talks about the future of bioanalytical science and technologies. Ian Jardine (Thermo Fisher Scientific) introduced new developments in Orbitrap high-resolution MS and its applications. As a core research tool for protein analysis, he presented examples of high-resolution MS applications for quantitation of peptides/proteins, characterization of peptides ('bottom up') and intact proteins ('top down') as well as quantitative cellular proteomics. As a routine high performance LC-MS/MS tool for small-molecule analysis, several examples were presented in the area of integrating biomarker discovery and reactive metabolite screening applications. There is great potential for high-resolution MS in clinical diagnostics and he presented examples in anatomical pathology and expression pathology. Jardine predicted that MS will play an increasing role as a portable device for the applications, such as point-of-care therapeutic drug monitoring through the integration with new sampling technologies and new work flows. Mike Lee (Milestone Development Services) closed the conference with his perspective on the future of the pharmaceutical industry. Within the context of historical precedent and global trends, his perspectives focused mainly on bioanalytical considerations regarding emerging technology, applications and workflows. He offered recent perspectives and benchmarks from recent Land O'Lakes Bioanalytical Conferences. In 2005, the industry recognized that new drug and biologic applications had significantly decreased while the cost of drug and product development had soared over the past decade. Thus, new models and paradigms (more with less) were implemented and greater attention was given to forecasting and prediction as well as biologics. By 2007 technology and culture were identified as key elements for such change. Lee then shared a unique, interactive graphical representation of the current world that uses novel software to convert international statistics into an easy to visualize output. There are emerging trends: with ultrahigh performance LC; the use of high-resolution, accurate mass as an opportunity to introduce a novel filter (selectivity) for quantitation; nanospray as the preferred ionization technology for the analysis of proteins and peptides; and the use of dried sample spots for offline processing (massively parallel, remote and offline) of samples to decouple and miniaturize the chemistries involved with sample and sample preparation. For bioanalysis to grow and keep up the pace with the global market developments, output must be able to be shared with participants who are not necessarily bioanalytical experts.

\section{Evening sessions}

During the Tuesday evening session, Zhenmin Liang (Roche) provided an overview of matrix effects in LC-MS bioanalysis and some of the common problems that this presents to all bioanalytical scientists. Matrix effects may lead to either suppression or, less commonly, enhancement of ionization. This likely occurs in every injection of analyte, but usually has no impact on the overall analysis if it does not occur in the 
retention regions of the analytes being measured. Ways to assess matrix effect were discussed that included a nonquantitative assessment aimed at identifying the components causing the problem. One practical suggestion was to prepare LLOQ quality controls in multiple lots of control plasma and assess the results obtained in their assay. Methods to measure these factors involved spiking experiments and post-column infusion of analyte. Examples of matrix effects from various methods of sample preparation were presented. A practical hint was not to use drug analogs as internal standards in LC-MS assays. Liang indicated that the best overall approach for how to solve matrix effects problem was from knowledge of the chemistry of the drug molecule and the separation science used in its quantitation.

The analytical investigators forum was held on Thursday evening and provided a platform for scientists from different organizations to present their research. Gert Hendriks (PRA International) discussed the validation of a generic photo-diode array (PDA) assay for stock stability assessment. Stock solution comparisons are commonly performed by LC-MS using methods optimized for the analyte of interest. Compound dependent, these methods involve sample dilution and may have relatively high variances. A generic HPLC-PDA based method detects every UV absorbing compound and has relatively low variance. The validation approach and use of the generic HPLC-PDA was shown to be flexible and of sufficient precision to investigate the stock stability of the majority of the compounds. Steve Wiltshire (Agilux Laboratories) presented the results of a study on the challenges and solutions to biomarker analysis. The assay was for the determination of selected endogenous bioactive fatty acid amides (arachidonyl ethanolamide [AEA], oleoyl ethanolamide, palmitoyl ethanolamide) in human plasma as a marker for the extent of inhibition of fatty acid amide hydrolase. The method utilized d8AEA as the surrogate standard for AEA, but showed that 4 AEA had an equivalent response to AEA, whereas d8AEA did not. The method also introduced leachables/ extractables during sample work-up with the primary source of the contamination attributed to O-rings on the liquid-liquid extraction sample tubes. Bernd Bruenner (Amgen) discussed selected challenges in regulated bioanalysis, including incurred sample reanalysis (ISR) and the observation of drug in control plasma samples. Overall ISR experience in both large and small molecules was a $95.5 \%$ pass rate and the failures were limited to small molecule assays only. Reasons for the ISR failures included:

- Utilization of compromised stock solution for the ISR run;

- Inability to consistently perform dilutions;

- Lack of sample homogeneity;

- Poor chromatography due to a bad column.

In each case an investigation identified the cause and following resolution, successful ISR runs were executed. Detection of test article (drug) in control group plasma samples occurred in two separate oral gavage toxicity studies in two species. In the final presentation of the session, Li Sun (Merck) discussed the development of ultra-sensitive LC-MS/MS methodologies for support of human microdosing studies. Microdosing is a strategy for introducing investigational drugs into humans with limited safety information because the dose is $1 / 100$ th or less of a pharmacologically active dose and allows an early read on human PK but requires a sensitive analytical method (1-2 pg/ml). Accelerator MS is often used but is nonselective and expensive. In the current study, effective sample preparation was used to remove interferences and allow analyte enrichment. Mobile phase modifiers also enhanced sensitivity and together with utilization of ultraperformance LC and negative ion electrospray MS/MS allowed for the development and validation of ultrasensitive LC-MS/MS methods for four investigational HIV integrase inhibitors. The methods successfully supported the human microdose studies.

\section{Workshops \& round table discussions}

Tuesday afternoon's session complemented the morning plenary session and featured case studies on using biomarkers and diagnostic kits during drug development. John Cheng (Pfizer) opened the session by providing an overview of flow cytometry (FACS) and examples of its use for immune response monitoring and proofof-mechanism verification. Cytokine assays described included a cytokine storm assay and an assay to measure serum cytokine levels in response to an immune enhancer in clinical development. FACS can also be used to do lymphocyte subset counting in the context of mechanistic and safety biomarkers. The talk concluded with two case studies where FACS assays were developed to define PK/pharmacodynamic relationships using a whole blood kinase assay and a 
receptor internalization assay. Hawazin Faruki (LabCorp) described the benefits and pitfalls of using diagnostic kits in clinical trials. Using IGF-1 as an example, the speaker highlighted how the lack of standardization in how kits are used can impact performance and reviewed sources of variability including: interference from high affinity binding proteins; complex formation; and lot-to-lot variability in reagents. Faruki provided a thorough review of factors to consider in developing a second-generation assay for clinical application and possible commercialization. In the final presentation, Martin Nemansky (PRA International) provided a review of an elegant ultra-high sensitivity biomarker assay using immuno-PCR. Imperacer technology was compared with other assay platforms including ELISA, MSD ${ }^{\circledR}$ and Gyros ${ }^{\mathrm{TM}}$ and applications in PKs, pharmacodynamics and clinical diagnostics (e.g., phosphorylated TAU and amyloid- $\beta$, for Alzheimer's disease). Nemansky provided a development paradigm for an ultra-high sensitivity immune-PCR cytokine assay with a resulting validated range of $0.059-6000 \mathrm{pg} / \mathrm{ml}$.

The Thursday afternoon workshop considered problems commonly encountered in daily laboratory work. Six themes were discussed:

" Ligand-binding assays;

- Qualification of bioanalytical reference standards;

- ISR;

" Intermittent carryover effects;

- Stability, assay range and non-specific binding;

- Daily laboratory operations.

Discussions were interactive, with many attendees gaining new understandings that could be added to their own laboratories.

Wednesday evening, following the poster session, informal round tables were held on several topics. These discussions and facilitators covered:
- Quantitative nature of biomarker assays (Lakshmi Amaravadi, Biogen Idec);

- Progress of LC-MS/MS technology in the bioanalysis of large molecules (Qin C Ji, Bristol-Myers Squibb);

- Recent perspectives on bioanalytical approaches to address metabolites in safety training (Hongying Gao and Eric Fluhler, Pfizer);

- Issues and opportunities for using e-notebooks (Michael Hayes, Novartis).

\section{Conclusion}

In comparison to larger international conventions and because of the informal, noncommercial and isolated setting, attendees have more time to meet each other, discuss their organizations, practices and the science. Evaluation results were very positive and a summary is presented on the aforementioned website. The 2012 program is currently being developed and will be posted on the University of Wisconsin's website when finished [2].

Financial \& competing interests disclosure

The authors have no relevant affiliations or financial involvement with any organization or entity with a financial interest in or financial conflict with the subject matter or materials discussed in the manuscript. This includes employment, consultancies, honoraria, stock ownership or options, expert testimony, grants or patents received or pending, or royalties.

No writing assistance was utilized in the production of this manuscript.

\section{Bibliography}

1 12th Annual Land O'Lakes Bioanalytical

Conference: Agency and Industry Perspectives on

Biomarkers, Bloodspots and Beyond.

http://ce.pharmacy.wisc.edu/

courseinfo/2011JulyLOL

2 University of Wisconsin website.

www.pharmacy.wisc.edu/esp 